\title{
Rational Orthogonal versus Real Orthogonal
}

\author{
Dragomir Ž. Djoković* Simone Severini ${ }^{\dagger} \quad$ Ferenc Szöllősi ${ }^{\ddagger}$
}

November 16, 2018

\begin{abstract}
The main question we raise here is the following one: given a real orthogonal $n \times n$ matrix $X$, is it true that there exists a rational orthogonal matrix $Y$ having the same zero-pattern? We conjecture that this is the case and prove it for $n \leq 5$. We also consider the related problem for symmetric orthogonal matrices.
\end{abstract}

Keywords and phrases: Real and rational orthogonal matrices, zero-patterns, combinatorial orthogonality.

\section{Introduction}

Let $X=\left[X_{i, j}\right]$ be an $m \times n$ matrix over any field. The zero-pattern of $X$, denoted by $\underline{X}=\left[\underline{X}_{i, j}\right]$, is the $m \times n(0,1)$-matrix such that

$$
\underline{X}_{i, j}= \begin{cases}1, & \text { if } X_{i, j} \neq 0 \\ 0, & \text { if } X_{i, j}=0\end{cases}
$$

We shall say that $\underline{X}$ is the support of $X$.

A square matrix $X$ is said to be unitary if its entries are complex and $X X^{\dagger}=I$, where $X^{\dagger}$ is the transpose conjugate of $X$ and $I$ is the identity matrix. A square matrix $X$ is said to be real orthogonal (or, equivalently, orthogonal) if its entries are real and $X X^{T}=I$, where $X^{T}$ is the transpose of $X$. A square matrix $X$ is said to be rational orthogonal if it is orthogonal and its entries are rational. The sets of unitary, orthogonal, and rational orthogonal matrices of size $n$ are denoted by $U(n), O(n)$ and $O_{n}(\mathbb{Q})$, respectively.

The notion and the study of the zero-patterns of unitary matrices go back to [5] (see also [4]) in the mathematical context, and to [11] (see also [10]), motivated by foundational

\footnotetext{
${ }^{*}$ Department of Pure Mathematics, University of Waterloo, Waterloo N2L 3G1, ON Canada; djokovic@uwaterloo.ca

${ }^{\dagger}$ Institute for Quantum Computing and Department of Combinatorics \& Optimization, University of Waterloo, Waterloo N2L 3G1, ON Canada; simoseve@gmail.com

${ }^{\ddagger}$ Institute of Mathematics and its Applications, Central European University (CEU), H-1051 Budapest, Nádor u. 9, Hungary; szoferi@gmail.com
} 
questions in quantum mechanics. An extended list of references on this topic is contained in [15]. For a comprehensive reference in matrix theory see, e.g., [8].

When discussing properties of zero-patterns, it is natural to ask whether the number field influences their structure. Specifically, in this paper we formulate and support the following two conjectures.

Conjecture 1 For any $X \in U(n)$ there exists $Y \in O(n)$ such that $\underline{X}=\underline{Y}$.

Conjecture 2 For any $Y \in O(n)$ there exists $Z \in O_{n}(\mathbb{Q})$ such that $\underline{Y}=\underline{Z}$.

Our main tool of analysis will be the notion of a strongly quadrangular matrix introduced in [14]. This extends naturally the concept of quadrangularity (or, equivalently, combinatorial orthogonality [1]). A matrix $X$ is said to be quadrangular if every two rows and every two columns "intersect" in more than a single entry whenever their intersection is nonempty. In other words, the inner product of every two rows and every two columns of $\underline{X}$ is not 1. Let $X=\left[X_{i, j}\right]$ be a complex $m \times n$ matrix. We write $X>0$ if all $X_{i, j}>0$. For $R \subseteq\{1,2, \ldots, m\}$ and $C \subseteq\{1,2, \ldots, n\}$, we denote by $X_{R}^{C}$ the $|R| \times|C|$ submatrix of $X$ in the intersection of the rows and the columns indexed by $R$ and $C$, respectively.

Definition 3 (Strongly quadrangular matrix) We say that an $m \times n\{0,1\}$-matrix $X=\left[X_{i, j}\right]$ is row strongly quadrangular (RSQ) if there does not exist $R \subseteq\{1,2, \ldots, m\}$ with $|R| \geq 2$ such that, defining $R^{\prime}=\left\{k: X_{i, k} X_{j, k}=1\right.$ for some $i \neq j$ in $\left.R\right\}$, we have $\left|R^{\prime}\right|<|R|$ and $X_{R}^{R^{\prime}}$ has no zero-rows. We say that an $m \times n\{0,1\}$-matrix $X$ is strongly quadrangular (SQ) if both $X$ and $X^{T}$ are $R S Q$.

In [14], it was proved that if $X \in U(n)$ then $\underline{X}$ is SQ, but the converse is not necessarily true (see also [12]). Proposition 4 below gives the smallest possible SQ zero-patterns that do not support unitary matrices.

So far we have been unable to exhibit counterexamples which would disprove Conjecture 1 or Conjecture 2. We can however get a feeling about the problem, by explicitly working out concrete situations. For instance, Beasley, Brualdi and Shader [1] have shown that if $X$ is a real matrix with zero-pattern

$$
\underline{X}=\left[\begin{array}{lllllllllll}
1 & 1 & 0 & 1 & 0 & 0 & 1 & 0 & 0 & 0 & 1 \\
1 & 1 & 1 & 0 & 1 & 0 & 0 & 1 & 0 & 0 & 0 \\
0 & 1 & 1 & 1 & 0 & 1 & 0 & 0 & 1 & 0 & 0 \\
0 & 0 & 1 & 1 & 1 & 0 & 1 & 0 & 0 & 1 & 0 \\
0 & 0 & 0 & 1 & 1 & 1 & 0 & 1 & 0 & 0 & 1 \\
1 & 0 & 0 & 0 & 1 & 1 & 1 & 0 & 1 & 0 & 0 \\
0 & 1 & 0 & 0 & 0 & 1 & 1 & 1 & 0 & 1 & 0 \\
0 & 0 & 1 & 0 & 0 & 0 & 1 & 1 & 1 & 0 & 1 \\
1 & 0 & 0 & 1 & 0 & 0 & 0 & 1 & 1 & 1 & 0 \\
0 & 1 & 0 & 0 & 1 & 0 & 0 & 0 & 1 & 1 & 1 \\
1 & 0 & 1 & 0 & 0 & 1 & 0 & 0 & 0 & 1 & 1
\end{array}\right]
$$


then $X \notin O(11)$. Once verified that that $\underline{X}$ is SQ, we observe that $X$ is not a candidate for a counterexample to Conjecture 1 , therefore corroborating the idea that the number field does not have a strong role in determining a zero-pattern. We can proceed as follows in four steps:

- By multiplying the columns 1,2,4,7, and 11 by suitable phase factors, all entries in the first row are real;

- By multiplying the rows $2,3,5,6,7,9,10$ and 11 by phase factors, the entries

$$
(2,1),(3,2),(5,4),(6,1),(7,2),(9,1),(10,2) \text { and }(11,1)
$$

are real;

- By multiplying the columns 3,5,6,8 and 9 by phase factors, the entries

$$
(2,3),(2,5),(2,8),(3,6) \text { and }(3,9)
$$

are real;

- Finally, by multiplying the rows 4 and 8 , and the column 10 by phase factors, the entries

$$
(4,3),(8,3) \text { and }(4,10)
$$

are real.

At this point, all the entries mentioned above are real. If $X \in U(n)$ then the inner products of different rows of the matrix obtained with these steps must vanish. It follows that $X$ is a real matrix, but we know that $X \notin O(11)$ by [1].

Here we adopt a systematic approach to our conjectures. In Section 2, we verify Conjecture 1 and Conjecture 2 for all $(0,1)$-matrices of size $n \leq 5$. For this purpose, we use the tables in [15] of all SQ $(0,1)$-matrices of small size. On the way, we prove that some of those are not zero-patterns of unitary matrices, thus refining the classification of [15]. In Section 3, we construct examples of symmetric rational orthogonal matrices with specified indecomposable zero-pattern and specified trace. In Section 4, we construct some infinite families of rational orthogonal matrices. The constructions are based on orthogonal designs, graphs and combinatorial arguments. We conclude our paper in Section 5 with three intriguing open problems.

Recall that an $n \times n$ matrix $X$ that contains an $s \times(n-s)$ zero submatrix for $0<s<n$ is said to be decomposable. If no such submatrix exists then $X$ is said to be indecomposable. 


\section{Rational orthogonal matrices of small size}

We shall consider the indecomposable SQ zero-patterns of size $n \leq 5$. Two $(0,1)$-matrices $X$ and $Y$ are said to be equivalent if there are permutation matrices $P$ and $Q$ such that $P X Q=Y$. A list of representatives for equivalence classes of indecomposable SQ zeropatterns of size $n \leq 5$ was drawn in [15]. We construct rational orthogonal matrices for each specific zero-pattern. This is not possible for the cases 14,15 and 16, because it turns out that those do not support unitary matrices. Here is a formal statement of such a fact:

Proposition 4 There is no matrix $X \in U(5)$ such that $\underline{X}$ is one of the following zeropatterns:

$$
\left[\begin{array}{lllll}
0 & 0 & 1 & 1 & 1 \\
0 & 0 & 1 & 1 & 1 \\
1 & 1 & 0 & 1 & 1 \\
1 & 1 & 1 & 1 & 1 \\
1 & 1 & 1 & 1 & 1
\end{array}\right]_{14},\left[\begin{array}{lllll}
0 & 0 & 1 & 1 & 1 \\
0 & 0 & 1 & 1 & 1 \\
1 & 1 & 0 & 1 & 1 \\
1 & 1 & 1 & 0 & 1 \\
1 & 1 & 1 & 1 & 1
\end{array}\right]_{15}, \quad\left[\begin{array}{lllll}
0 & 0 & 1 & 1 & 1 \\
0 & 0 & 1 & 1 & 1 \\
1 & 1 & 0 & 1 & 1 \\
1 & 1 & 1 & 0 & 1 \\
1 & 1 & 1 & 1 & 0
\end{array}\right]_{16} .
$$

Proof. Suppose that such $X$ exists. Let $Y=X_{\{3,4,5\}}^{\{1,2\}}$ and $Z=X_{\{3,4,5\}}^{\{3,4\}}$. By inspecting the above three zero-patterns, we conclude that $Z$ has rank 2. Since $X$ is unitary, we have $Y^{\dagger} Z=0$ and so the two columns of $Y$ must be linearly dependent. Consequently, the first two columns of $X$ are linearly dependent, which is a contradiction.

The main result of the paper is essentially the following theorem:

Theorem 5 Conjecture 1 and Conjecture 2 are true for $n \leq 5$.

Proof. Clearly it suffices to consider only the indecomposable SQ zero-patterns. For $n \leq 5$, these zero-patterns have been enumerated in [15] (up to equivalence). All of these zero-patterns support unitary matrices, except the three cases for $n=5$ mentioned in Proposition 4. Thus, in order to prove the theorem it suffices to construct a matrix in $O_{n}(\mathbb{Q})$ for each of the remaining zero-patterns. This is done in the list below. Many of these matrices have been constructed by using an exhaustive search but in some cases this was not possible and we have resorted to ad hoc methods. Some of these methods are sketched in Section 4.

In our list, a matrix $X$ will be written in the form

$$
X=\frac{1}{d}\left[\begin{array}{ccc}
* & \cdots & * \\
\vdots & \ddots & \vdots \\
* & \cdots & *
\end{array}\right]_{k}
$$


where $k$ is simply a numerical label for the equivalence classes of zero-patterns, identical to the labels in [15]. We say that the denominator $d$ is minimal if it is the smallest possible denominator among all rational orthogonal matrices with the same zero-pattern. All denominators in the list are minimal except for a few cases, when $n=5$. Exceptions are the cases $6,7,19,28$, and 31 .

$2.1 \quad n=2$

$$
\frac{1}{5}\left[\begin{array}{rr}
3 & 4 \\
4 & -3
\end{array}\right]_{1}
$$

$2.2 n=3$

$$
\frac{1}{25}\left[\begin{array}{rrr}
16 & 12 & 15 \\
12 & 9 & -20 \\
15 & -20 & 0
\end{array}\right]_{1} \quad \frac{1}{3}\left[\begin{array}{rrr}
2 & -1 & 2 \\
-1 & 2 & 2 \\
2 & 2 & -1
\end{array}\right]_{2}
$$

$2.3 n=4$

$$
\begin{aligned}
& \frac{1}{9}\left[\begin{array}{rrrr}
8 & -3 & 2 & 2 \\
-3 & 0 & 6 & 6 \\
2 & 6 & -4 & 5 \\
2 & 6 & 5 & -4
\end{array}\right]_{1} \quad \frac{1}{9}\left[\begin{array}{rrrr}
6 & 0 & 3 & -6 \\
\mathbf{0} & 1 & 8 & 4 \\
3 & 8 & -2 & 2 \\
-6 & 4 & 2 & -5
\end{array}\right]_{2} \\
& \frac{1}{33}\left[\begin{array}{rrrr}
16 & 7 & \mathbf{0} & -28 \\
7 & \mathbf{0} & 32 & 4 \\
\mathbf{0} & 32 & -1 & 8 \\
-28 & 4 & 8 & -15
\end{array}\right]_{3} \quad \frac{1}{3}\left[\begin{array}{rrrr}
1 & 2 & -2 & \mathbf{0} \\
2 & \mathbf{0} & 1 & 2 \\
-2 & 1 & \mathbf{0} & 2 \\
\mathbf{0} & 2 & 2 & -1
\end{array}\right]_{4} \\
& \frac{1}{65}\left[\begin{array}{rrrr}
25 & \mathbf{0} & -36 & 48 \\
\mathbf{0} & \mathbf{0} & 52 & 39 \\
-36 & 52 & -9 & 12 \\
48 & 39 & 12 & -16
\end{array}\right]_{5} \quad \frac{1}{15}\left[\begin{array}{rrrr}
\mathbf{0} & \mathbf{0} & 9 & 12 \\
2 & 14 & -4 & 3 \\
10 & -5 & -8 & 6 \\
11 & 2 & 8 & -6
\end{array}\right]_{6} \\
& \frac{1}{25}\left[\begin{array}{rrrr}
15 & \mathbf{0} & 12 & 16 \\
\mathbf{0} & 20 & 12 & -9 \\
-20 & \mathbf{0} & 9 & 12 \\
\mathbf{0} & 15 & -16 & 12
\end{array}\right]_{7} \quad \frac{1}{2}\left[\begin{array}{rrrr}
1 & 1 & 1 & -1 \\
1 & 1 & -1 & 1 \\
1 & -1 & 1 & 1 \\
-1 & 1 & 1 & 1
\end{array}\right]_{8} \text {. }
\end{aligned}
$$


$2.4 n=5$

$$
\begin{aligned}
& \frac{1}{4}\left[\begin{array}{rrrrr}
3 & 1 & 1 & 1 & -2 \\
1 & 3 & -1 & -1 & 2 \\
1 & -1 & 3 & -1 & 2 \\
1 & -1 & -1 & 3 & 2 \\
-2 & 2 & 2 & 2 & 0
\end{array}\right]_{1} \quad \frac{1}{7}\left[\begin{array}{rrrrr}
4 & -3 & 2 & 4 & 2 \\
-3 & 4 & 2 & 4 & 2 \\
2 & 2 & 3 & -4 & 4 \\
4 & 4 & -4 & 1 & 0 \\
2 & 2 & 4 & 0 & -5
\end{array}\right]_{2} \\
& \frac{1}{11}\left[\begin{array}{rrrrr}
8 & 4 & 1 & 2 & -6 \\
4 & 5 & -4 & \mathbf{0} & 8 \\
1 & -4 & \mathbf{0} & 10 & 2 \\
2 & \mathbf{0} & 10 & -1 & 4 \\
-6 & 8 & 2 & 4 & -1
\end{array}\right]_{3} \quad \frac{1}{5}\left[\begin{array}{rrrrr}
3 & 2 & -2 & 2 & 2 \\
2 & 2 & 1 & -4 & \mathbf{0} \\
-2 & 1 & 2 & \mathbf{0} & 4 \\
2 & -4 & \mathbf{0} & -1 & 2 \\
2 & \mathbf{0} & 4 & 2 & -1
\end{array}\right]_{4} \\
& \frac{1}{147}\left[\begin{array}{rrrrr}
145 & 8 & \mathbf{0} & 14 & -18 \\
8 & 51 & 80 & -112 & \mathbf{0} \\
\mathbf{0} & 80 & 47 & 70 & 90 \\
14 & -112 & 70 & \mathbf{0} & 63 \\
-18 & \mathbf{0} & 90 & 63 & -96
\end{array}\right]_{5} \quad \frac{1}{625}\left[\begin{array}{rrrrr}
256 & 240 & 192 & 375 & 300 \\
240 & 225 & 180 & \mathbf{0} & -500 \\
192 & 180 & 144 & -500 & 225 \\
375 & \mathbf{0} & -500 & \mathbf{0} & \mathbf{0} \\
300 & -500 & 225 & \mathbf{0} & \mathbf{0}
\end{array}\right]_{6} \\
& \frac{1}{75}\left[\begin{array}{rrrrr}
50 & -25 & \mathbf{0} & 30 & 40 \\
-25 & 50 & \mathbf{0} & 30 & 40 \\
\mathbf{0} & \mathbf{0} & \mathbf{0} & 60 & -45 \\
30 & 30 & 60 & -9 & -12 \\
40 & 40 & -45 & -12 & -16
\end{array}\right]_{7} \quad \frac{1}{25}\left[\begin{array}{rrrrr}
16 & 12 & \mathbf{0} & 12 & -9 \\
12 & 9 & \mathbf{0} & -16 & 12 \\
\mathbf{0} & \mathbf{0} & \mathbf{0} & 15 & 20 \\
12 & -16 & 15 & \mathbf{0} & \mathbf{0} \\
-9 & 12 & 20 & \mathbf{0} & \mathbf{0}
\end{array}\right]_{8} \\
& \frac{1}{9}\left[\begin{array}{rrrrr}
8 & 2 & 2 & \mathbf{0} & -3 \\
2 & 4 & -6 & 3 & 4 \\
2 & -6 & 1 & 6 & 2 \\
\mathbf{0} & 3 & 6 & \mathbf{0} & 6 \\
-3 & 4 & 2 & 6 & -4
\end{array}\right]_{9} \quad \frac{1}{9}\left[\begin{array}{rrrrr}
\mathbf{0} & \mathbf{0} & 6 & 3 & 6 \\
\mathbf{0} & 4 & 5 & 2 & -6 \\
6 & -5 & \mathbf{0} & 4 & -2 \\
3 & 6 & -4 & 4 & 2 \\
6 & 2 & 2 & -6 & 1
\end{array}\right]_{10} \\
& \frac{1}{27}\left[\begin{array}{rrrrr}
20 & -12 & 10 & 9 & 2 \\
-12 & 6 & 15 & 18 & \mathbf{0} \\
10 & 15 & 2 & \mathbf{0} & -20 \\
9 & 18 & \mathbf{0} & \mathbf{0} & 19 \\
2 & \mathbf{0} & -20 & 18 & -21
\end{array}\right]_{11} \quad \frac{1}{21}\left[\begin{array}{rrrrr}
\mathbf{0} & \mathbf{0} & 4 & 5 & 20 \\
\mathbf{0} & 7 & 10 & 16 & -6 \\
18 & -10 & \mathbf{0} & 4 & -1 \\
9 & 16 & -10 & \mathbf{0} & 2 \\
6 & 6 & 15 & -12 & \mathbf{0}
\end{array}\right]_{12} \\
& \frac{1}{9}\left[\begin{array}{rrrrr}
4 & 4 & 2 & 6 & 3 \\
4 & 4 & 2 & -3 & -6 \\
2 & 2 & 1 & -6 & 6 \\
6 & -3 & -6 & \mathbf{0} & \mathbf{0} \\
3 & -6 & 6 & \mathbf{0} & \mathbf{0}
\end{array}\right]_{13} \quad \frac{1}{9}\left[\begin{array}{rrrrr}
8 & \mathbf{0} & -3 & 2 & 2 \\
\mathbf{0} & 7 & \mathbf{0} & 4 & -4 \\
-3 & \mathbf{0} & \mathbf{0} & 6 & 6 \\
2 & 4 & 6 & -3 & 4 \\
2 & -4 & 6 & 4 & -3
\end{array}\right]_{17}
\end{aligned}
$$




$$
\begin{aligned}
& \frac{1}{9}\left[\begin{array}{rrrrr}
5 & 4 & \mathbf{0} & -6 & 2 \\
4 & 3 & -4 & 6 & 2 \\
\mathbf{0} & -4 & 1 & \mathbf{0} & 8 \\
-6 & 6 & \mathbf{0} & \mathbf{0} & 3 \\
2 & 2 & 8 & 3 & \mathbf{0}
\end{array}\right]_{18} \quad \frac{1}{441}\left[\begin{array}{rrrrr}
400 & \mathbf{0} & 100 & 105 & 116 \\
\mathbf{0} & 400 & 80 & 84 & -145 \\
100 & 80 & 41 & -420 & \mathbf{0} \\
105 & 84 & -420 & \mathbf{0} & \mathbf{0} \\
116 & -145 & \mathbf{0} & \mathbf{0} & -400
\end{array}\right]_{19} \\
& \frac{1}{6}\left[\begin{array}{rrrrr}
3 & 3 & 3 & -3 & \mathbf{0} \\
3 & 3 & -3 & 3 & \mathbf{0} \\
3 & -3 & 1 & 1 & 4 \\
-3 & 3 & 1 & 1 & 4 \\
\mathbf{0} & \mathbf{0} & 4 & 4 & -2
\end{array}\right]_{20}^{1} \quad \frac{1}{21}\left[\begin{array}{rrrrr}
18 & \mathbf{0} & 6 & \mathbf{0} & -9 \\
\mathbf{0} & 17 & 6 & -10 & 4 \\
6 & 6 & \mathbf{0} & 15 & 12 \\
\mathbf{0} & -10 & 15 & -4 & 10 \\
-9 & 4 & 12 & 10 & -10
\end{array}\right]_{21}^{1} \\
& \frac{1}{15}\left[\begin{array}{rrrrr}
10 & \mathbf{0} & 3 & 4 & 10 \\
\mathbf{0} & 10 & 6 & 8 & -5 \\
3 & 6 & 6 & -12 & \mathbf{0} \\
4 & 8 & -12 & -1 & \mathbf{0} \\
10 & -5 & \mathbf{0} & \mathbf{0} & -10
\end{array}\right]_{22} \quad \frac{1}{5}\left[\begin{array}{rrrrr}
3 & 2 & 2 & -2 & -2 \\
2 & 3 & -2 & 2 & 2 \\
2 & -2 & 3 & -2 & 3 \\
-2 & 2 & 2 & -2 & 3 \\
-2 & 2 & 2 & 3 & -2
\end{array}\right]_{23} \\
& \frac{1}{11}\left[\begin{array}{rrrrr}
\mathbf{0} & \mathbf{0} & 2 & 6 & 9 \\
1 & -2 & -6 & 8 & -4 \\
2 & 7 & 6 & 4 & -4 \\
4 & -8 & 6 & 1 & -2 \\
10 & 2 & -3 & -2 & 2
\end{array}\right]_{24} \quad \frac{1}{10}\left[\begin{array}{rrrrr}
\mathbf{0} & \mathbf{0} & \mathbf{0} & 6 & 8 \\
1 & -7 & -5 & -4 & 3 \\
1 & -7 & 5 & 4 & -3 \\
7 & 1 & -5 & 4 & -3 \\
7 & 1 & 5 & -4 & 3
\end{array}\right]_{25} \\
& \frac{1}{45}\left[\begin{array}{rrrrr}
\mathbf{0} & \mathbf{0} & \mathbf{0} & 27 & 36 \\
\mathbf{0} & 42 & 6 & 12 & -9 \\
5 & -16 & 12 & 32 & -24 \\
20 & 2 & -39 & 8 & -6 \\
40 & 1 & 18 & -8 & 6
\end{array}\right]_{26} \quad \frac{1}{45}\left[\begin{array}{rrrrr}
\mathbf{0} & \mathbf{0} & \mathbf{0} & 27 & -36 \\
\mathbf{0} & 35 & 20 & 16 & 12 \\
15 & \mathbf{0} & -30 & 24 & 18 \\
30 & -20 & 25 & 8 & 6 \\
30 & 20 & -10 & -20 & -15
\end{array}\right]_{27} \\
& \frac{1}{165}\left[\begin{array}{rrrrr}
\mathbf{0} & \mathbf{0} & \mathbf{0} & 132 & -99 \\
\mathbf{0} & 80 & 35 & 84 & 112 \\
5 & \mathbf{0} & 160 & -24 & -32 \\
160 & -35 & \mathbf{0} & 12 & 16 \\
-40 & -140 & 20 & 45 & 60
\end{array}\right]_{28} \quad \frac{1}{39}\left[\begin{array}{rrrrr}
\mathbf{0} & \mathbf{0} & \mathbf{0} & 15 & 36 \\
28 & 5 & -6 & 24 & -10 \\
2 & 18 & -32 & -12 & 5 \\
27 & -4 & 10 & -24 & 10 \\
-2 & 34 & 19 & \mathbf{0} & \mathbf{0}
\end{array}\right]_{29} \\
& \frac{1}{15}\left[\begin{array}{rrrrr}
\mathbf{0} & \mathbf{0} & \mathbf{0} & 9 & 12 \\
\mathbf{0} & 14 & 2 & 4 & -3 \\
10 & -4 & 3 & 8 & -6 \\
10 & 3 & 4 & -8 & 6 \\
5 & 2 & -14 & \mathbf{0} & \mathbf{0}
\end{array}\right]_{30} \quad \frac{1}{165}\left[\begin{array}{rrrrr}
\mathbf{0} & \mathbf{0} & \mathbf{0} & 99 & 132 \\
\mathbf{0} & 35 & 140 & 64 & -48 \\
160 & \mathbf{0} & -20 & 28 & -21 \\
40 & 20 & 75 & -112 & 84 \\
5 & -160 & 40 & \mathbf{0} & \mathbf{0}
\end{array}\right]_{31}
\end{aligned}
$$




$$
\begin{aligned}
& \frac{1}{15}\left[\begin{array}{rrrrr}
\mathbf{0} & \mathbf{0} & \mathbf{0} & 9 & 12 \\
\mathbf{0} & 10 & 10 & 4 & -3 \\
10 & \mathbf{0} & -5 & 8 & -6 \\
10 & 5 & \mathbf{0} & -8 & 6 \\
5 & -10 & 10 & \mathbf{0} & \mathbf{0}
\end{array}\right]_{32} \quad \frac{1}{21}\left[\begin{array}{rrrrr}
\mathbf{0} & \mathbf{0} & 7 & 14 & 14 \\
\mathbf{0} & 7 & 0 & 14 & -14 \\
-6 & 18 & 6 & -6 & 3 \\
9 & 8 & -16 & 2 & 6 \\
18 & 2 & 10 & -3 & -2
\end{array}\right]_{33} \\
& \frac{1}{21}\left[\begin{array}{rrrrr}
\mathbf{0} & \mathbf{0} & 6 & 18 & 9 \\
\mathbf{0} & 14 & \mathbf{0} & -7 & 14 \\
13 & 8 & 12 & \mathbf{0} & -8 \\
4 & 10 & -15 & 8 & -6 \\
16 & -9 & -6 & -2 & 8
\end{array}\right]_{34} \quad \frac{1}{21}\left[\begin{array}{rrrrr}
\mathbf{0} & \mathbf{0} & 6 & 9 & 18 \\
\mathbf{0} & 14 & \mathbf{0} & -14 & 7 \\
20 & 1 & 6 & \mathbf{0} & -2 \\
4 & 10 & -15 & 10 & \mathbf{0} \\
-5 & 12 & 12 & 8 & -8
\end{array}\right]_{35} \\
& \frac{1}{15}\left[\begin{array}{rrrrr}
\mathbf{0} & \mathbf{0} & \mathbf{0} & 9 & 12 \\
\mathbf{0} & -12 & -9 & \mathbf{0} & \mathbf{0} \\
5 & -6 & 8 & -8 & 6 \\
10 & -3 & 4 & 8 & -6 \\
10 & 6 & -8 & -4 & 3
\end{array}\right]_{36} \quad \frac{1}{13}\left[\begin{array}{rrrrr}
\mathbf{0} & \mathbf{0} & 4 & 3 & 12 \\
1 & 10 & \mathbf{0} & -8 & 2 \\
2 & 7 & 6 & 8 & -4 \\
8 & 2 & -9 & 4 & 2 \\
-10 & 4 & -6 & 4 & 1
\end{array}\right]_{37} \\
& \frac{1}{15}\left[\begin{array}{rrrrr}
\mathbf{0} & \mathbf{0} & 5 & -10 & 10 \\
3 & -12 & \mathbf{0} & 6 & 6 \\
12 & 1 & 8 & \mathbf{0} & -4 \\
-6 & 4 & 10 & 8 & 3 \\
6 & 8 & -6 & 5 & 8
\end{array}\right]_{38} \quad \frac{1}{33}\left[\begin{array}{rrrrr}
\mathbf{0} & \mathbf{0} & 11 & 22 & 22 \\
8 & 15 & \mathbf{0} & -20 & 20 \\
10 & 12 & 26 & \mathbf{0} & -13 \\
30 & -12 & -6 & 3 & \mathbf{0} \\
5 & 24 & -16 & 14 & -6
\end{array}\right]_{39} \\
& \frac{1}{65}\left[\begin{array}{rrrrr}
\mathbf{0} & \mathbf{0} & \mathbf{0} & 25 & 60 \\
-7 & 60 & 24 & \mathbf{0} & \mathbf{0} \\
24 & -20 & 57 & \mathbf{0} & \mathbf{0} \\
36 & 9 & -12 & -48 & 20 \\
48 & 12 & -16 & 36 & -15
\end{array}\right]_{40} \quad \frac{1}{75}\left[\begin{array}{rrrrr}
\mathbf{0} & \mathbf{0} & \mathbf{0} & 45 & 60 \\
\mathbf{0} & \mathbf{0} & 60 & 36 & -27 \\
-10 & 70 & 15 & -16 & 12 \\
50 & 25 & -30 & 32 & -24 \\
55 & -10 & 30 & -32 & 24
\end{array}\right]_{41} \\
& \frac{1}{65}\left[\begin{array}{rrrrr}
\mathbf{0} & \mathbf{0} & \mathbf{0} & 39 & 52 \\
\mathbf{0} & \mathbf{0} & 25 & -48 & 36 \\
25 & -60 & \mathbf{0} & \mathbf{0} & \mathbf{0} \\
36 & 15 & 48 & 16 & -12 \\
48 & 20 & -36 & -12 & 9
\end{array}\right]_{42} \quad \frac{1}{125}\left[\begin{array}{rrrrr}
\mathbf{0} & \mathbf{0} & \mathbf{0} & 75 & 100 \\
\mathbf{0} & 75 & 100 & \mathbf{0} & \mathbf{0} \\
\mathbf{0} & 60 & -45 & 80 & -60 \\
100 & -48 & 36 & 36 & -27 \\
75 & 64 & -48 & -48 & 36
\end{array}\right]_{43}
\end{aligned}
$$

\section{Symmetric rational orthogonal matrices}

A square matrix $X$ is involutory if $X^{2}=I$. It is well known that a matrix $X \in U(n)$ is hermitian if and only if it is involutory. In particular, a matrix $X \in O(n)$ is symmetric if and only if it is involutory. 
One can easily formulate the symmetric analogues of Conjecture 1 and Conjecture 2 . For the sake of simplicity we shall formulate just the combined conjecture.

Conjecture 6 For any hermitian $X \in U(n)$ there exists a symmetric $Z \in O_{n}(\mathbb{Q})$ such that $\underline{X}=\underline{Z}$ and $\operatorname{Tr}(X)=\operatorname{Tr}(Z)$.

Let $X=X^{\dagger} \in U(n)$. Then $X^{2}=I_{n}$ and so the eigenvalues of $X$ belong to $\{ \pm 1\}$. Consequently, $\operatorname{Tr}(X)$ is an integer congruent to $n \bmod 2$. Since $-X=\underline{X}$ and $\operatorname{Tr}(-X)=$ $-\operatorname{Tr}(X)$, in proving this conjecture we may assume that $\operatorname{Tr}(X) \geq 0$. Clearly, we can also assume that the zero-pattern $\underline{X}$ is indecomposable (we also know that it is necessarily SQ). There are further restrictions on possible values of the trace.

Proposition 7 There is no indecomposable hermitian matrix $X \in U(n), n \geq 2$, with $X_{1,2}=0$ and $\operatorname{Tr}(X)=n-2$.

Proof. Suppose that such a matrix, $X$, exists. As $X^{2}=I$ and $X \neq \pm I$, the eigenvalues of $X$ are +1 and -1 and the two eigenspaces of $X$ are orthogonal to each other. By indecomposability we have $X_{2,2} \neq 1$. Let $\left\{e_{1}, \ldots, e_{n}\right\}$ be the standard basis of $\mathbb{C}^{n}$. Since $X_{1,2}=0$, the vector $X e_{2}$ is orthogonal to $e_{1}$ and also $X e_{2} \neq e_{2}$. Thus the vector $v=$ $X e_{2}-e_{2}$ is nonzero and $v \perp e_{1}$. As $X v=-v$ and the -1-eigenspace of $X$ is 1-dimensional, we conclude that the subspace $v^{\perp}$ is the +1 -eigenspace of $X$. Hence $X e_{1}=e_{1}$, i.e., $X_{1,1}=1$. This contradicts the indecomposability of $X$.

The objective of this section is to provide a support for the above conjecture by constructing examples of symmetric rational orthogonal matrices with specified indecomposable zero-pattern and specified trace. We shall consider zero-patterns of size $n \leq 5$.

Two symmetric $(0,1)$-matrices $X$ and $Y$ are said to be congruent if there is a permutation matrix $P$ such that $P X P^{T}=Y$. In graph-theoretical terms, the permutation matrix $P$ represents an isomorphism between the undirected graph with adjacency matrix $X$ and the undirected graph with adjacency matrix $Y$.

Let $X=\left[X_{i, j}\right] \in U(n)$ be a hermitian matrix. We say that $X$ is in quasi-normal form if $\operatorname{Tr}(X) \geq 0$ and $X_{1,1} \geq X_{2,2} \geq \cdots \geq X_{n, n}$. In our list a matrix $X$ will be written in the form

$$
X=\frac{1}{d}\left[\begin{array}{ccc}
* & \cdots & * \\
\vdots & \ddots & \vdots \\
* & \cdots & *
\end{array}\right]_{k, l}^{t},
$$

where $k$ and $l$ are simply numerical labels and $t$ is the trace of the matrix. The index $k$ corresponds to the one used for the matrices in Section 2. The index $l$ specifies the congruence class of symmetric zero-patterns within the $k$-th equivalence class.

Our list is not complete. We are in fact unable to construct symmetric rational orthogonal matrices with specified trace for exactly two among all zero-patterns. For these 
matrices, we give examples of matrices as close as possible to symmetric rational one in Section 5. All denominators in the list are minimal except for a few cases, when $n=5$. Exceptions are the cases $(k, l)=(2,2),(3,2),(6,1),(11,2),(22,2)$.

$3.1 n=2$

$$
\frac{1}{5}\left[\begin{array}{rr}
3 & 4 \\
4 & -3
\end{array}\right]_{1}^{0}
$$

$3.2 n=3$

$$
\frac{1}{25}\left[\begin{array}{rrr}
16 & 12 & 15 \\
12 & 9 & -20 \\
15 & -20 & 0
\end{array}\right]_{1}^{1} \quad \frac{1}{3}\left[\begin{array}{rrr}
2 & -1 & 2 \\
-1 & 2 & 2 \\
2 & 2 & -1
\end{array}\right]_{2}^{1}
$$

$3.3 n=4$

$$
\begin{aligned}
& \frac{1}{9}\left[\begin{array}{rrrr}
8 & -3 & 2 & 2 \\
-3 & 0 & 6 & 6 \\
2 & 6 & -4 & 5 \\
2 & 6 & 5 & -4
\end{array}\right]_{1}^{0} \quad \frac{1}{9}\left[\begin{array}{rrrr}
8 & 2 & -2 & 3 \\
2 & 5 & 4 & -6 \\
-2 & 4 & 5 & 6 \\
3 & -6 & 6 & 0
\end{array}\right]_{1}^{2} \\
& \frac{1}{9}\left[\begin{array}{rrrr}
6 & \mathbf{0} & 3 & -6 \\
\mathbf{0} & 1 & 8 & 4 \\
3 & 8 & -2 & 2 \\
-6 & 4 & 2 & -5
\end{array}\right]_{2,1}^{0} \quad \frac{1}{9}\left[\begin{array}{rrrr}
8 & 5 & -10 & 6 \\
5 & \mathbf{0} & 10 & 10 \\
-10 & 10 & \mathbf{0} & 5 \\
6 & 10 & 5 & -8
\end{array}\right]_{2,2}^{0} \\
& \frac{1}{33}\left[\begin{array}{rrrr}
16 & 7 & \mathbf{0} & -28 \\
7 & \mathbf{0} & 32 & 4 \\
\mathbf{0} & 32 & -1 & 8 \\
-28 & 4 & 8 & -15
\end{array}\right]_{3}^{0} \quad \frac{1}{3}\left[\begin{array}{rrrr}
2 & \mathbf{0} & 2 & 1 \\
\mathbf{0} & 2 & 1 & -2 \\
2 & 1 & -2 & \mathbf{0} \\
1 & -2 & \mathbf{0} & -2
\end{array}\right]_{4,1}^{0} \\
& \frac{1}{3}\left[\begin{array}{rrrr}
1 & 2 & -2 & \mathbf{0} \\
2 & \mathbf{0} & 1 & 2 \\
-2 & 1 & \mathbf{0} & 2 \\
\mathbf{0} & 2 & 2 & -1
\end{array}\right]_{4,2}^{0} \quad \frac{1}{65}\left[\begin{array}{rrrr}
25 & \mathbf{0} & -36 & 48 \\
\mathbf{0} & \mathbf{0} & 52 & 39 \\
-36 & 52 & -9 & 12 \\
48 & 39 & 12 & -16
\end{array}\right]_{5}^{0} \\
& \frac{1}{2}\left[\begin{array}{rrrr}
1 & -1 & 1 & 1 \\
-1 & 1 & 1 & 1 \\
1 & 1 & -1 & 1 \\
1 & 1 & 1 & -1
\end{array}\right]_{8}^{0} \quad \frac{1}{2}\left[\begin{array}{rrrr}
1 & 1 & 1 & -1 \\
1 & 1 & -1 & 1 \\
1 & -1 & 1 & 1 \\
-1 & 1 & 1 & 1
\end{array}\right]_{8}^{2} \text {. }
\end{aligned}
$$


$3.4 n=5$

$$
\begin{aligned}
& \frac{1}{4}\left[\begin{array}{rrrrr}
3 & 1 & 1 & 1 & -2 \\
1 & 3 & -1 & -1 & 2 \\
1 & -1 & 3 & -1 & 2 \\
1 & -1 & -1 & 3 & 2 \\
-2 & 2 & 2 & 2 & 0
\end{array}\right]_{1}^{3} \quad \frac{1}{27}\left[\begin{array}{rrrrr}
19 & 4 & -12 & 12 & 8 \\
4 & 16 & 6 & -15 & 14 \\
-12 & 6 & 9 & 18 & 12 \\
12 & -15 & 18 & 0 & 6 \\
8 & 14 & 12 & 6 & -17
\end{array}\right]_{1}^{1} \\
& \frac{1}{7}\left[\begin{array}{rrrrr}
4 & -3 & 2 & 4 & 2 \\
-3 & 4 & 2 & 4 & 2 \\
2 & 2 & 3 & -4 & 4 \\
4 & 4 & -4 & 1 & \mathbf{0} \\
2 & 2 & 4 & \mathbf{0} & -5
\end{array}\right]_{2,1}^{1} \quad \frac{1}{375}\left[\begin{array}{rrrrr}
200 & 90 & 120 & 125 & -250 \\
90 & 168 & -276 & 150 & 75 \\
120 & -276 & 7 & 200 & 100 \\
125 & 150 & 200 & \mathbf{0} & 250 \\
-250 & 75 & 100 & 250 & \mathbf{0}
\end{array}\right]_{2,2}^{1} \\
& \frac{1}{11}\left[\begin{array}{rrrrr}
8 & 4 & 1 & 2 & -6 \\
4 & 5 & -4 & \mathbf{0} & 8 \\
1 & -4 & \mathbf{0} & 10 & 2 \\
2 & \mathbf{0} & 10 & -1 & 4 \\
-6 & 8 & 2 & 4 & -1
\end{array}\right]_{3,1}^{1} \quad \frac{1}{325}\left[\begin{array}{rrrrr}
245 & 84 & 80 & -140 & 112 \\
84 & 80 & 112 & 35 & -280 \\
80 & 112 & \mathbf{0} & 280 & 91 \\
-140 & 35 & 280 & \mathbf{0} & 80 \\
112 & -280 & 91 & 80 & \mathbf{0}
\end{array}\right]_{3,2}^{1} \\
& \frac{1}{5}\left[\begin{array}{rrrrr}
3 & 2 & -2 & 2 & 2 \\
2 & 2 & 1 & -4 & \mathbf{0} \\
-2 & 1 & 2 & \mathbf{0} & 4 \\
2 & -4 & \mathbf{0} & -1 & 2 \\
2 & \mathbf{0} & 4 & 2 & -1
\end{array}\right]_{4,1}^{1} \quad \frac{1}{5}\left[\begin{array}{rrrrr}
4 & \mathbf{0} & 1 & -2 & 2 \\
\mathbf{0} & 4 & -2 & 1 & 2 \\
1 & -2 & \mathbf{0} & 4 & 2 \\
-2 & 1 & 4 & \mathbf{0} & 2 \\
2 & 2 & 2 & 2 & -3
\end{array}\right]_{4,2}^{1} \\
& \frac{1}{147}\left[\begin{array}{rrrrr}
145 & 8 & \mathbf{0} & 14 & -18 \\
8 & 51 & 80 & -112 & \mathbf{0} \\
\mathbf{0} & 80 & 47 & 70 & 90 \\
14 & -112 & 70 & \mathbf{0} & 63 \\
-18 & \mathbf{0} & 90 & 63 & -96
\end{array}\right]_{5,1}^{1} \quad \frac{1}{625}\left[\begin{array}{rrrrr}
256 & 240 & 192 & 375 & 300 \\
240 & 225 & 180 & \mathbf{0} & -500 \\
192 & 180 & 144 & -500 & 225 \\
375 & \mathbf{0} & -500 & \mathbf{0} & \mathbf{0} \\
300 & -500 & 225 & \mathbf{0} & \mathbf{0}
\end{array}\right]_{6}^{1} \\
& \frac{1}{75}\left[\begin{array}{rrrrr}
50 & -25 & \mathbf{0} & 30 & 40 \\
-25 & 50 & \mathbf{0} & 30 & 40 \\
\mathbf{0} & \mathbf{0} & \mathbf{0} & 60 & -45 \\
30 & 30 & 60 & -9 & -12 \\
40 & 40 & -45 & -12 & -16
\end{array}\right]_{7}^{1} \quad \frac{1}{25}\left[\begin{array}{ccccc}
16 & 12 & \mathbf{0} & 12 & -9 \\
12 & 9 & \mathbf{0} & -16 & 12 \\
\mathbf{0} & \mathbf{0} & \mathbf{0} & 15 & 20 \\
12 & -16 & 15 & \mathbf{0} & \mathbf{0} \\
-9 & 12 & 20 & \mathbf{0} & \mathbf{0}
\end{array}\right]_{8}^{1} \\
& \frac{1}{9}\left[\begin{array}{rrrrr}
8 & 2 & 2 & \mathbf{0} & -3 \\
2 & 4 & -6 & 3 & 4 \\
2 & -6 & 1 & 6 & 2 \\
\mathbf{0} & 3 & 6 & \mathbf{0} & 6 \\
-3 & 4 & 2 & 6 & -4
\end{array}\right]_{9}^{1} \quad \frac{1}{27}\left[\begin{array}{rrrrr}
19 & 12 & 8 & -12 & 4 \\
12 & 5 & -20 & 12 & 4 \\
8 & -20 & 3 & \mathbf{0} & 16 \\
-12 & 12 & \mathbf{0} & \mathbf{0} & 21 \\
4 & 4 & 16 & 21 & \mathbf{0}
\end{array}\right]_{10}^{1}
\end{aligned}
$$




$$
\begin{aligned}
& \frac{1}{27}\left[\begin{array}{rrrrr}
20 & -12 & 10 & 9 & 2 \\
-12 & 6 & 15 & 18 & \mathbf{0} \\
10 & 15 & 2 & \mathbf{0} & -20 \\
9 & 18 & \mathbf{0} & \mathbf{0} & 18 \\
2 & \mathbf{0} & -20 & 18 & -1
\end{array}\right]_{11,1}^{1} \\
& \frac{1}{78625}\left[\begin{array}{rrrrr}
50320 & 27156 & \mathbf{0} & -46620 & 27183 \\
27156 & 28305 & -43680 & 51408 & 9620 \\
\mathbf{0} & -43680 & \mathbf{0} & 12025 & 64260 \\
-46620 & 51408 & 12025 & \mathbf{0} & 34944 \\
27183 & 9620 & 64260 & 34944 & \mathbf{0}
\end{array}\right]_{11,2}^{1} \\
& \frac{1}{9}\left[\begin{array}{rrrrr}
4 & 4 & 2 & 6 & 3 \\
4 & 4 & 2 & -3 & -6 \\
2 & 2 & 1 & -6 & 6 \\
6 & -3 & -6 & \mathbf{0} & \mathbf{0} \\
3 & -6 & 6 & \mathbf{0} & \mathbf{0}
\end{array}\right]_{13}^{1} \quad \frac{1}{9}\left[\begin{array}{rrrrr}
8 & \mathbf{0} & -3 & 2 & 2 \\
\mathbf{0} & 7 & \mathbf{0} & 4 & -4 \\
-3 & \mathbf{0} & \mathbf{0} & 6 & 6 \\
2 & 4 & 6 & -3 & 4 \\
2 & -4 & 6 & 4 & -3
\end{array}\right]_{17}^{1} \\
& \frac{1}{9}\left[\begin{array}{rrrrr}
5 & 4 & \mathbf{0} & -6 & 2 \\
4 & 3 & -4 & 6 & 2 \\
\mathbf{0} & -4 & 1 & \mathbf{0} & 8 \\
-6 & 6 & \mathbf{0} & \mathbf{0} & 3 \\
2 & 2 & 8 & 3 & \mathbf{0}
\end{array}\right]_{18}^{1} \quad \frac{1}{441}\left[\begin{array}{rrrrr}
400 & \mathbf{0} & 100 & 105 & 116 \\
\mathbf{0} & 400 & 80 & 84 & -145 \\
100 & 80 & 41 & -420 & \mathbf{0} \\
105 & 84 & -420 & \mathbf{0} & \mathbf{0} \\
116 & -145 & \mathbf{0} & \mathbf{0} & -400
\end{array}\right]_{19}^{1} \\
& \frac{1}{6}\left[\begin{array}{rrrrr}
3 & 3 & 3 & -3 & \mathbf{0} \\
3 & 3 & -3 & 3 & \mathbf{0} \\
3 & -3 & 1 & 1 & 4 \\
-3 & 3 & 1 & 1 & 4 \\
\mathbf{0} & \mathbf{0} & 4 & 4 & -2
\end{array}\right]_{20}^{1} \quad \frac{1}{21}\left[\begin{array}{rrrrr}
18 & \mathbf{0} & 6 & \mathbf{0} & -9 \\
\mathbf{0} & 17 & 6 & -10 & 4 \\
6 & 6 & \mathbf{0} & 15 & 12 \\
\mathbf{0} & -10 & 15 & -4 & 10 \\
-9 & 4 & 12 & 10 & -10
\end{array}\right]_{21}^{1} \\
& \frac{1}{15}\left[\begin{array}{rrrrr}
10 & \mathbf{0} & 3 & 4 & 10 \\
\mathbf{0} & 10 & 6 & 8 & -5 \\
3 & 6 & 6 & -12 & \mathbf{0} \\
4 & 8 & -12 & -1 & \mathbf{0} \\
10 & -5 & \mathbf{0} & \mathbf{0} & -10
\end{array}\right]_{22,1}^{1} \quad \frac{1}{75}\left[\begin{array}{rrrrr}
39 & \mathbf{0} & -48 & 30 & 30 \\
\mathbf{0} & 25 & \mathbf{0} & -50 & 50 \\
-48 & \mathbf{0} & 11 & 40 & 40 \\
30 & -50 & 40 & \mathbf{0} & 25 \\
30 & 50 & 40 & 25 & \mathbf{0}
\end{array}\right]_{22,2}^{1} \\
& \frac{1}{5}\left[\begin{array}{rrrrr}
3 & 2 & 2 & -2 & -2 \\
2 & 3 & -2 & 2 & 2 \\
2 & -2 & 3 & -2 & 2 \\
-2 & 2 & 2 & -2 & 3 \\
-2 & 2 & 2 & 3 & -2
\end{array}\right]_{23}^{1} \quad \frac{1}{5}\left[\begin{array}{rrrrr}
3 & 2 & 2 & 2 & -2 \\
2 & 3 & -2 & -2 & 2 \\
2 & -2 & 3 & -2 & 2 \\
2 & -2 & -2 & 3 & 2 \\
-2 & 2 & 2 & 2 & 3
\end{array}\right]_{23}^{3}
\end{aligned}
$$




\section{Infinite families of rational orthogonal matrices}

In this section we employ different techniques to construct infinite families of symmetric rational orthogonal matrices with specified zero-pattern and trace. The following wellknown fact will be useful. We include a proof for the sake of completeness.

Proposition 8 The set $S O_{n}(\mathbb{Q})$ is dense in $S O(n)$ (in Euclidean topology).

Proof. It is sufficient to observe that the Cayley transformation

$$
X \longmapsto Y=\frac{I+X}{I-X},
$$

from the space of $n \times n$ real skew-symmetric matrices to $S O(n)$ has dense image, and if $X$ is a rational matrix so is $Y$.

Let $\Delta_{n, k}$ be the $n \times n$ zero-pattern all of whose entries are 1 except for the first $k$ diagonal entries which are 0 .

Corollary 9 Let $0 \leq k<l<n$. If there exists $X=X^{T} \in O_{n}(\mathbb{Q})$ with $\underline{X}=\Delta_{n, l}$, then there exists $Y=Y^{T} \in O_{n}(\mathbb{Q})$ with $\underline{X}=\Delta_{n, k}$ and $\operatorname{Tr}(X)=\operatorname{Tr}(Y)$.

Proof. Without any loss of generality we may assume that $l=k+1$. Let $X=X^{T} \in O_{n}(\mathbb{Q})$ be such that $\underline{X}=\Delta_{n, l}$. Set $Y=P \times P^{T}$, where $P=I_{k} \oplus R \oplus I_{n-l-1}$ and $R$ is the rotation matrix

$$
\left[\begin{array}{rr}
\cos \theta & -\sin \theta \\
\sin \theta & \cos \theta
\end{array}\right]
$$

Clearly we can choose $\theta \in \mathbb{R}$ such that $\underline{Y}=\Delta_{n, k}$. Since the rational points on the unit circle are dense (see Proposition 8), we can replace $R$ with $R_{1} \in S O_{2}(\mathbb{Q})$ without affecting the zero-pattern of $Y$.

\subsection{Symmetric rational orthogonal matrices with few zero entries}

Observe that the matrix $X_{n}=I_{n}-\frac{2}{n} J_{n}$ is rational orthogonal and involutory, where $J_{n}$ denotes the all-ones matrix. Moreover, $\operatorname{Tr}\left(X_{n}\right)=n-2$ and, if $n>2, X_{n}$ has no zero entries, i.e., $\underline{X}=J_{n}$. This $X_{n}$ is often called Grover matrix in the literature of quantum computation (see, e.g., [13]).

Proposition 10 Let $t=n-2 k$ where $k \in\{1,2, \ldots, n-1\}$. Then there exists a symmetric matrix $X \in O_{n}(\mathbb{Q})$ such that $\operatorname{Tr}(X)=t$ and $\underline{X}=J_{n}$. 
Proof. Noe that the assertion is vacuous for $n=1$ and trivial for $n=2$. We proceed by induction on $n \geq 3$. We may assume that $t \geq 0$. If $k=1$ the above observation shows that the assertion is true. Let $k>1$. Then $t=n-2 k \leq n-4$ implies that $n \geq 4$. By induction hypothesis there exists a symmetric matrix $Y \in O_{n-2}(\mathbb{Q})$ such that $\operatorname{Tr}(Y)=t$ and $\underline{Y}=J_{n-2}$. The matrix

$$
Z=Y \oplus \frac{1}{5}\left[\begin{array}{rr}
3 & 4 \\
4 & -3
\end{array}\right] \in O_{n}(\mathbb{Q})
$$

is symmetric with $\operatorname{Tr}(Z)=t$. By using Proposition 8 , we can choose $P \in O_{n}(\mathbb{Q})$ such that $X=P Z P^{T}$ has no zero entries, i.e., $\underline{X}=J_{n}$.

Proposition 11 Let $X=X^{T} \in O_{n}(\mathbb{Q})$ be such that $X_{i, n} \neq 0$ for $1 \leq i \leq n$. Then, $m>n>1$, there exists $Y=Y^{T} \in O_{m}(\mathbb{Q})$ such that $X_{i, j}=0$ if and only if $Y_{i, j}=0$, for $1 \leq i, j \leq n$, and $Y_{i, j} \neq 0$, for $i>n$. Moreover, $Y$ can be chosen so that $\operatorname{Tr}(Y)=$ $m-n+\operatorname{Tr}(X)$.

Proof. Without any loss of generality we may assume that $m=n+1$. Then we can take $Y=P(X \oplus[1]) P^{T}$, where

$$
P=I_{n-1} \oplus\left[\begin{array}{rr}
a & b \\
b & -a
\end{array}\right]
$$

and $a, b \in \mathbb{Q}^{*}$ are chosen such that $a^{2}+b^{2}=1$ and $a^{2} / b^{2} \neq-X_{n, n}^{ \pm 1}$.

By using the fact that $\Delta_{4,2}$ supports a matrix $X=X^{T} \in O_{4}(\mathbb{Q})$ such that $\operatorname{Tr}(X)=0$, if follows from the above proposition that $\Delta_{m, 2}, m \geq 4$, supports a matrix $Y=Y^{T} \in O_{m}(\mathbb{Q})$ with $\operatorname{Tr}(Y)=m-4$.

\subsection{Symmetric rational orthogonal matrices with zero-pattern $J_{n}-I_{n}$}

If there exists a symmetric matrix $X \in O(n)$ with zero-pattern $J_{n}-I_{n}$, then $n$ must be even. Indeed since such $X$ is involutory, its trace is an integer of the same parity as $n$.

A conference matrix of order $n$ is an $n \times n$ matrix $C$ with zero diagonal and all other entries in $\{ \pm 1\}$ and such that $C C^{T}=(n-1) I_{n}$. If a conference matrix of order $n>1$ exists, then $n$ must be even. It is known that they exist for all even orders $n=2 m \leq 64$ except for $m=11,17$ and 29 (when they do not exist). A conference matrix is normalized if all entries in the first row and column are equal to 1 , except the $(1,1)$ entry which is 0 .

Let $C$ be a normalized conference matrix of order $n$. If $n \equiv 2(\bmod 4)$, then $C$ is necessarily symmetric. On the other hand, if $n \equiv 0(\bmod 4)$, then the submatrix of $C$ obtained by deleting the first row and column is necessarily skew-symmetric. By a well known construction of Paley (see, e.g. [7]), we know that there exist conference matrices of order $n=1+p^{k}$ for any odd prime $p$ and any positive integer $k$. From these facts we deduce the following result. 
Proposition 12 Let $C$ be a normalized conference matrix of order $n=1+m^{2}$, where $m$ is an odd positive integer. Then $\frac{1}{m} C$ is a symmetric rational orthogonal matrix with zero-pattern $J_{n}-I_{n}$. Such $C$ exists if $m$ is an odd prime power.

\subsection{Symmetric rational orthogonal matrices from orthogonal designs}

An orthogonal design (see, e.g., [6]) of order $n$ and type $\left(s_{1}, s_{2}, \ldots, s_{u}\right)$ for $s_{i}>0$, on the commuting variables $x_{1}, x_{2}, \ldots, x_{u}$, is an $n \times n$ matrix $M$ with entries from $\left\{0, \pm x_{i}: i=\right.$ $1,2, \ldots, u\}$ such that

$$
M M^{T}=\left(\sum_{i=1}^{u} s_{i} x_{i}^{2}\right) I_{n} .
$$

Such design can be used to construct infinitely many rational orthogonal matrices with the same zero-pattern. As an example, consider the following orthogonal design:

$$
\begin{aligned}
X=\left[\begin{array}{rrrrrrrr}
x & y & z & 0 & a & 0 & 0 & -b \\
y & -x & 0 & -z & 0 & -a & b & 0 \\
z & 0 & -x & y & 0 & -b & -a & 0 \\
0 & -z & y & x & b & 0 & 0 & a \\
a & 0 & 0 & b & -x & y & z & 0 \\
0 & -a & -b & 0 & y & x & 0 & -z \\
0 & b & -a & 0 & z & 0 & x & y \\
-b & 0 & 0 & a & 0 & -z & y & -x
\end{array}\right], \\
X X^{T}=\left(x^{2}+y^{2}+z^{2}+a^{2}+b^{2}\right) I .
\end{aligned}
$$

If we set $x=y=z=1 / 4, a=1 / 2$, and $b=3 / 4$, we then obtain a symmetric matrix in $\mathrm{O}_{8}(\mathbb{Q})$, with the same zero-pattern as $X$.

\subsection{Indecomposable rational orthogonal matrices with maximal number of zero entries}

We recall from [1] that the maximum number of the zero entries in an indecomposable $n \times n$ unitary matrix, $n \geq 2$, is $(n-2)^{2}$. Let us say that an indecomposable $n \times n$ zero-pattern is maximal if it has exactly $(n-2)^{2}$ zero entries. In the same paper it is shown that, for $n \geq 5$, the maximal zero-patterns form either a single equivalence class or two equivalence classes which are transposes of each other. We shall see below that both possibilities occur. It is also known (see [2]) that the number of zero entries in indecomposable $n \times n$ unitary matrices can take any of the values $0,1,2, \ldots,(n-2)^{2}$.

We shall use the special zigzag matrices introduced in [3]. These are the matrices $X$ 
defined by means of two sequences $x_{0}, x_{1}, x_{2}, \ldots$ and $y_{1}, y_{2}, \ldots$ as follows:



If the above sequences are infinite, $X$ will be an infinite matrix and we shall denote it by $X_{\infty}$. If $X$ is of size $n$ then we shall denote it by $X_{n}$. Thus $X_{n}$ is defined by two finite sequences: $x_{0}, x_{1}, \ldots, x_{n}$ and $y_{1}, y_{2}, \ldots, y_{n-1}$. Note that $X_{n}$ is just the $n \times n$ submatrix lying in the left upper corner of $X_{\infty}$. If $x_{k}^{2}+y_{k}^{2}=1$ for $1 \leq k \leq n-1$ and $x_{0}, x_{n} \in\{ \pm 1\}$, then $X_{n} \in O(n)$.

Proposition 13 If $\underline{M}$ is a maximal $n \times n$ zero-pattern then there exists $X \in O_{n}(\mathbb{Q})$ such that $\underline{X}=\underline{M}$.

Proof. In the above matrix $X_{n}$, we can chose the rational values for $x_{k}$ and $y_{k}$ such that $x_{k}^{2}+y_{k}^{2}=1$ and $x_{k} y_{k} \neq 0$, for $1 \leq k \leq n-1$, and set $x_{0}=x_{n}=1$. Then $X_{n} \in O_{n}(\mathbb{Q})$ and it has the desired zero-pattern. It remains to observe that $\underline{X_{n}}$ must be equivalent to $\underline{M}$ or $\underline{M}^{T}$ by a result of $[1]$.

Let $Y_{n}$ be the matrix obtained from $X_{n}$ by reversing the order of its rows. Let us denote its zero-pattern by $\Lambda_{n}$. This is an example of a maximal zero-pattern (see [1]).

We set $x_{0}=x_{n}=1$ and impose the conditions $x_{k}=x_{n-k}, y_{k}=y_{n-k}$ and $x_{k}^{2}+y_{k}^{2}=1$ for $1 \leq k<n$. We can choose such $x_{k}$ and $y_{k}$ to be rational and nonzero. Hence, in that case maximal zero-patterns form just one equivalence class. If $n$ is odd, then $\Lambda_{n}$ and $Y_{n}$ are symmetric matrices. On the other hand, if $n$ is even then $\Lambda_{n}$ is not symmetric. In fact, in that case the equivalence class of $\Lambda_{n}$ contains no symmetric patterns. This follows by comparing the row sums and column sums of $\Lambda_{n}$. For $n=6$, we have verified that the maximal zero-patterns form two equivalence classes.

Now let $n=2 m$ be even. Denote by $\Lambda_{n}^{\#}$ the $n \times n$ symmetric zero-pattern in the following infinite sequence:

$$
\Lambda_{4}^{\#}=\left[\begin{array}{llll}
1 & 1 & 1 & 1 \\
1 & 1 & 1 & 1 \\
1 & 1 & 1 & \mathbf{0} \\
1 & 1 & \mathbf{0} & \mathbf{0}
\end{array}\right], \quad \Lambda_{6}^{\#}=\left[\begin{array}{lllllll}
\mathbf{0} & \mathbf{0} & \mathbf{0} & \mathbf{0} & 1 & 1 \\
\mathbf{0} & 1 & 1 & 1 & 1 & 1 \\
\mathbf{0} & 1 & 1 & 1 & 1 & 1 \\
\mathbf{0} & 1 & 1 & 1 & \mathbf{0} & \mathbf{0} \\
& 1 & 1 & \mathbf{0} & \mathbf{0} & \mathbf{0} \\
1 & 1 & 1 & \mathbf{0} & \mathbf{0} & \mathbf{0}
\end{array}\right]
$$




$$
\begin{aligned}
\Lambda_{8}^{\#}=\left[\begin{array}{lllll|llll}
\mathbf{0} & \mathbf{0} & \mathbf{0} & \mathbf{0} & \mathbf{0} & 1 & 1 & 1 \\
\mathbf{0} & \mathbf{0} & \mathbf{0} & \mathbf{0} & \mathbf{0} & 1 & 1 & 1 \\
& \mathbf{0} & \mathbf{0} & 1 & 1 & 1 & 1 & 1 & \mathbf{0} \\
\mathbf{0} & \mathbf{0} & 1 & 1 & 1 & 1 & 1 & \mathbf{0} \\
\mathbf{0} & \mathbf{0} & 1 & 1 & 1 & \mathbf{0} & \mathbf{0} & \mathbf{0} \\
& 1 & 1 & 1 & 1 & \mathbf{0} & \mathbf{0} & \mathbf{0} & \mathbf{0} \\
\\
1 & 1 & 1 & 1 & \mathbf{0} & \mathbf{0} & \mathbf{0} & \mathbf{0} & \\
1 & 1 & \mathbf{0} & \mathbf{0} & \mathbf{0} & \mathbf{0} & \mathbf{0} & \mathbf{0} &
\end{array}\right], \\
\Lambda_{10}^{\#}=\left[\begin{array}{lllllllllll}
\mathbf{0} & \mathbf{0} & \mathbf{0} & \mathbf{0} & \mathbf{0} & \mathbf{0} & \mathbf{0} & \mathbf{0} & 1 & 1 \\
\mathbf{0} & \mathbf{0} & \mathbf{0} & \mathbf{0} & \mathbf{0} & \mathbf{0} & 1 & 1 & 1 & 1 \\
\mathbf{0} & \mathbf{0} & \mathbf{0} & \mathbf{0} & \mathbf{0} & \mathbf{0} & 1 & 1 & 1 & 1 \\
\mathbf{0} & \mathbf{0} & \mathbf{0} & 1 & 1 & 1 & 1 & 1 & \mathbf{0} & \mathbf{0} \\
\mathbf{0} & \mathbf{0} & \mathbf{0} & 1 & 1 & 1 & 1 & 1 & \mathbf{0} & \mathbf{0} \\
\mathbf{0} & \mathbf{0} & \mathbf{0} & 1 & 1 & 1 & \mathbf{0} & \mathbf{0} & \mathbf{0} & \mathbf{0} \\
\mathbf{0} & 1 & 1 & 1 & 1 & \mathbf{0} & \mathbf{0} & \mathbf{0} & \mathbf{0} & \mathbf{0} \\
\mathbf{0} & 1 & 1 & 1 & 1 & \mathbf{0} & \mathbf{0} & \mathbf{0} & \mathbf{0} & \mathbf{0} \\
\hdashline 1 & 1 & 1 & \mathbf{0} & \mathbf{0} & \mathbf{0} & \mathbf{0} & \mathbf{0} & \mathbf{0} & \mathbf{0} \\
1 & 1 & 1 & \mathbf{0} & \mathbf{0} & \mathbf{0} & \mathbf{0} & \mathbf{0} & \mathbf{0} & \mathbf{0}
\end{array}\right], \ldots
\end{aligned}
$$

Note that $\Lambda_{n}^{\#}$ has exactly $4 n-3$ ones.

Theorem 14 For odd (resp. even) $n>2$ there exist symmetric rational orthogonal matrices with zero-pattern $\Lambda_{n}$ (resp. $\left.\Lambda_{n}^{\#}\right)$.

Proof. We have already taken care of the odd case. In the even case, we shall construct the required matrices $Z_{n}$ for $n=4,6$ and 8 only. It will be obvious how to proceed for bigger values of $n$. For $\left(x_{k}, y_{k}\right), k \geq 0$, we can choose any rational point on the unit circle $x^{2}+y^{2}=1$ such that $x_{k} y_{k} \neq 0$. For $n=4,6$ we take the matrices in the forms

$$
\begin{gathered}
Z_{4}=\left[\begin{array}{rrrrr}
x_{0} x_{1}^{2} & x_{0} x_{1} y_{1} & y_{0} x_{1} & y_{1} \\
x_{0} x_{1} y_{1} & x_{0} y_{1}^{2} & y_{0} y_{1} & -x_{1} \\
y_{0} x_{1} & y_{0} y_{1} & -x_{0} & \mathbf{0} \\
y_{1} & -x_{1} & \mathbf{0} & \mathbf{0}
\end{array}\right], \\
Z_{6}=\left[\begin{array}{rrrrrr}
\mathbf{0} & \mathbf{0} & \mathbf{0} & \mathbf{0} & y_{2} & x_{2} \\
\mathbf{0} & x_{0} x_{1}^{2} & x_{0} x_{1} y_{1} & y_{0} x_{1} & y_{1} x_{2} & -y_{1} y_{2} \\
\mathbf{0} & x_{0} x_{1} y_{1} & x_{0} y_{1}^{2} & y_{0} y_{1} & -x_{1} x_{2} & x_{1} y_{2} \\
\mathbf{0} & y_{0} x_{1} & y_{0} y_{1} & -x_{0} & \mathbf{0} & \mathbf{0} \\
y_{2} & y_{1} x_{2} & -x_{1} x_{2} & \mathbf{0} & \mathbf{0} & \mathbf{0} \\
x_{2} & -y_{1} y_{2} & x_{1} y_{2} & \mathbf{0} & \mathbf{0} & \mathbf{0}
\end{array}\right] .
\end{gathered}
$$


For $n=8$ we take

$$
Z_{8}=\left[\begin{array}{rrrrrrrr}
\mathbf{0} & \mathbf{0} & \mathbf{0} & \mathbf{0} & \mathbf{0} & y_{2} x_{3} & x_{2} x_{3} & y_{3} \\
\mathbf{0} & \mathbf{0} & \mathbf{0} & \mathbf{0} & \mathbf{0} & y_{2} y_{3} & x_{2} y_{3} & -x_{3} \\
\mathbf{0} & \mathbf{0} & x_{0} x_{1}^{2} & x_{0} x_{1} y_{1} & y_{0} x_{1} & y_{1} x_{2} & -y_{1} y_{2} & \mathbf{0} \\
\mathbf{0} & \mathbf{0} & x_{0} x_{1} y_{1} & x_{0} y_{1}^{2} & y_{0} y_{1} & -x_{1} x_{2} & x_{1} y_{2} & \mathbf{0} \\
\mathbf{0} & \mathbf{0} & y_{0} x_{1} & y_{0} y_{1} & -x_{0} & \mathbf{0} & \mathbf{0} & \mathbf{0} \\
y_{2} x_{3} & y_{2} y_{3} & y_{1} x_{2} & -x_{1} x_{2} & \mathbf{0} & \mathbf{0} & \mathbf{0} & \mathbf{0} \\
x_{2} x_{3} & x_{2} y_{3} & -y_{1} y_{2} & x_{1} y_{2} & \mathbf{0} & \mathbf{0} & \mathbf{0} & \mathbf{0} \\
y_{3} & -x_{3} & \mathbf{0} & \mathbf{0} & \mathbf{0} & \mathbf{0} & \mathbf{0} & \mathbf{0}
\end{array}\right] .
$$

In each of these cases $Z_{n}$ is orthogonal and symmetric, and so involutory matrix, with zero-pattern $\Lambda_{n}^{\#}$. In general, the construction can be best understood by considering the infinite matrix

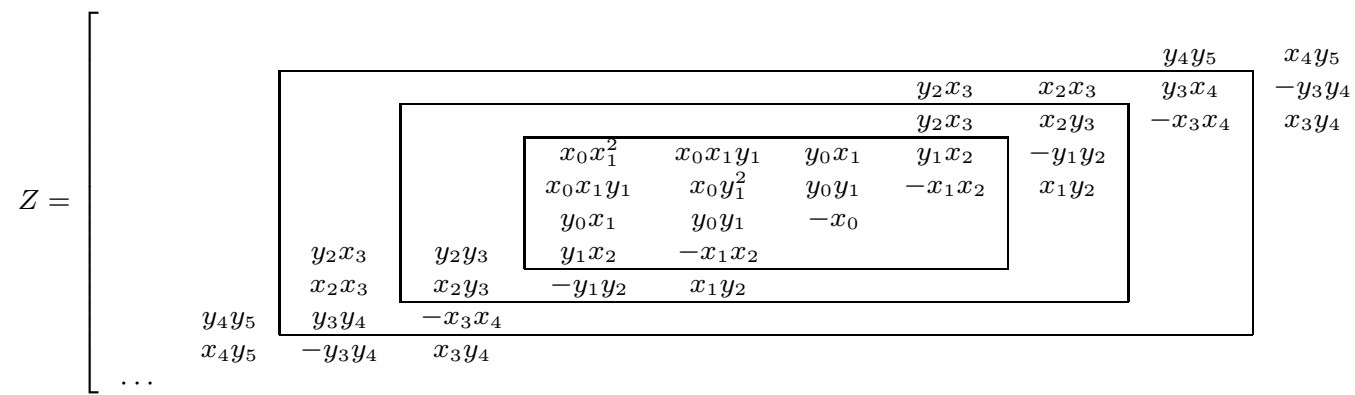

\subsection{Symmetric rational orthogonal matrices from hypercubes}

The Hamming distance between two words $v, w \in\{0,1\}^{n}$, denoted by $d(v, w)$, is the number of coordinates in which the words differ: $d(v, w):=\sum_{i=1}^{n}\left|v_{i}-w_{i}\right|$. The $n$ dimensional hypercube, denoted by $Q_{n}$, is the graph defined as follows: $V\left(Q_{n}\right)=\{0,1\}^{n}$; $\{v, w\} \in E\left(Q_{n}\right)$ if and only $d(v, w)=1$. The adjacency matrix of $Q_{n}$ can be constructed recursively:

$$
M\left(Q_{1}\right)=\left(\begin{array}{ll}
0 & 1 \\
1 & 0
\end{array}\right), \quad M\left(Q_{n}\right)=\left(\begin{array}{cc}
M\left(Q_{n-1}\right) & I \\
I & M\left(Q_{n-1}\right)
\end{array}\right), \quad \text { for } n \geq 2
$$

It is simple to verify that the following matrices are real orthogonal:

$$
M_{1}=\left[\begin{array}{cc}
0 & -1 \\
1 & 0
\end{array}\right], \quad M_{n}=\frac{1}{\sqrt{n}}\left[\begin{array}{cc}
M_{n-1} & -I \\
I & M_{n-1}^{-1}
\end{array}\right], \quad \text { for } n \geq 2 .
$$

Clearly, $\underline{M_{n}}=M\left(Q_{n}\right)$. 
Proposition 15 For each $n \geq 2$, there exist many infinitely symmetric matrices $Y_{n} \in$ $O_{2^{n}}(\mathbb{Q})$ such that $\underline{Y_{n}}=M\left(Q_{n}\right)$.

Proof. Let $x_{1}, x_{2}, \ldots$ be indeterminates. We define the matrices $X_{n}$ recursively by

$$
X_{1}=\left[\begin{array}{cc}
0 & x_{1} \\
x_{1} & 0
\end{array}\right], \quad X_{n}=\left[\begin{array}{cc}
X_{n-1} & x_{n} I \\
x_{n} I & -X_{n-1}
\end{array}\right], \quad \text { for } n \geq 2 .
$$

Note that $X_{n}^{T}=X_{n}$ and one can easily verify by induction that

$$
X_{n}^{2}=\left(x_{1}^{2}+x_{2}^{2}+\cdots+x_{n}^{2}\right) I_{2^{n}} .
$$

For a given $n \geq 1$, we choose nonzero rational numbers $\alpha_{1}, \alpha_{2}, \ldots, \alpha_{n}$ such that $\alpha_{1}^{2}+\alpha_{2}^{2}+$ $\cdots+\alpha_{n}^{2}=1$. Then if we set $x_{k}=\alpha_{k}(k=1,2, \ldots, n)$ in $X_{n}$, we obtain a symmetric rational orthogonal matrix $Y_{n}$ with $\underline{Y_{n}}=M\left(Q_{n}\right)$.

\subsection{Hessenberg rational orthogonal matrices}

Let $H_{n}$ be the lower triangular $n \times n$ Hessenberg zero-pattern:

$$
H_{n}=\left[\begin{array}{cccccc}
1 & 1 & 1 & 1 & \cdots & 1 \\
1 & 1 & 1 & 1 & & 1 \\
0 & 1 & 1 & 1 & & \vdots \\
& 0 & 1 & 1 & & \\
& & \ddots & \ddots & & \\
0 & & & 0 & & 1
\end{array}\right]
$$

We consider here the corresponding symmetric zero-pattern $S_{n} H_{n}$, where

$$
S_{n}=\left[\begin{array}{llll}
\mathbf{0} & & & 1 \\
& & 1 & \\
& \ldots & & \\
1 & & & \mathbf{0}
\end{array}\right]
$$

is the antidiagonal permutation matrix.

Proposition 16 There exist infinitely many $Y_{n}=Y_{n}^{T} \in O_{n}(\mathbb{Q})$ with $\underline{Y_{n}}=S_{n} H_{n}$.

Proof. Let $a, b \in \mathbb{Q}$ be nonzero and such that $a^{2}+b^{2}=1$. We define recursively the matrices $X_{n} \in O_{n}(\mathbb{Q}), n \geq 2$, by

$$
X_{2}=\left[\begin{array}{cc}
a & -b \\
b & a
\end{array}\right]
$$




$$
X_{n}=\left[\begin{array}{cc}
X_{n-1} & \mathbf{0} \\
\mathbf{0} & 1
\end{array}\right]\left[\begin{array}{cc}
I_{n-2} & \mathbf{0} \\
\mathbf{0} & X_{2}
\end{array}\right]
$$

for $n \geq 3$. The matrices $Y_{n}=S_{n} H_{n}$ are symmetric and satisfy $\underline{Y_{n}}=S_{n} H_{n}$. Indeed, we have

$$
\begin{aligned}
Y_{2}=\left[\begin{array}{rr}
b & a \\
a & -b
\end{array}\right], \quad Y_{3}=\left[\begin{array}{rrr}
\mathbf{0} & b & a \\
b & a^{2} & -a b \\
a & -a b & b^{2}
\end{array}\right] \\
Y_{4}=\left[\begin{array}{rrrr}
\mathbf{0} & \mathbf{0} & b & a \\
\mathbf{0} & b & a^{2} & -a b \\
b & a^{2} & -a^{2} b & a b^{2} \\
a & -a b & a b^{2} & -b^{3}
\end{array}\right], \text { and } Y_{5}=\left[\begin{array}{rrrrr}
\mathbf{0} & \mathbf{0} & \mathbf{0} & b & a \\
\mathbf{0} & \mathbf{0} & b & a^{2} & -a b \\
\mathbf{0} & b & a^{2} & -a^{2} b & a b^{2} \\
b & a^{2} & -a^{2} b & a^{2} b^{2} & -a b^{3} \\
a & -a b & a b^{2} & -a b^{3} & b^{4}
\end{array}\right] .
\end{aligned}
$$

By induction on $n$ one can prove that

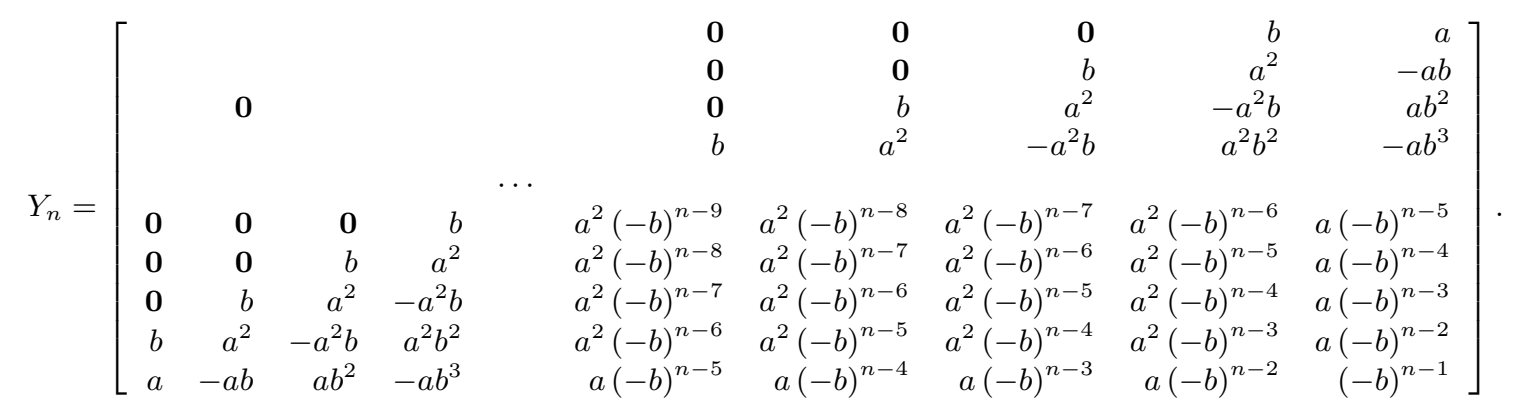

Note that the trace of $Y_{n}$ is zero for $n$ even and one for $n$ odd.

\section{Open problems}

In addition to the conjectures formulated in the paper, we state here some further open problems.

The first problem is of purely combinatorial nature.

Problem 17 Let $X$ be an $n \times n$ zero-pattern and assume that $X^{T}$ is equivalent to $X$. Is it true that $X$ is equivalent to a symmetric pattern?

We have verified that the answer to the above problem is affirmative for $n \leq 5$. 
Problem 18 Are there symmetric rational orthogonal matrices with the following zeropatterns:

$$
\left[\begin{array}{lllll}
1 & \mathbf{0} & 1 & 1 & 1 \\
\mathbf{0} & 1 & 1 & 1 & 1 \\
1 & 1 & \mathbf{0} & 1 & 1 \\
1 & 1 & 1 & \mathbf{0} & 1 \\
1 & 1 & 1 & 1 & \mathbf{0}
\end{array}\right] \quad, \quad\left[\begin{array}{lllll}
1 & 1 & 1 & 1 & \mathbf{0} \\
1 & 1 & 1 & \mathbf{0} & 1 \\
1 & 1 & \mathbf{0} & 1 & 1 \\
1 & \mathbf{0} & 1 & \mathbf{0} & 1 \\
\mathbf{0} & 1 & 1 & 1 & 1
\end{array}\right] ?
$$

As mentioned in Section 3, in spite of much effort we were not able to construct such matrices. Below we give some examples of matrices with the same zero-pattern as close as possible to be rational. For the first zero-patten we give two examples. The first one minimizes the denominator:

$$
\begin{gathered}
\frac{1}{4}\left[\begin{array}{rrrrr}
2 & \mathbf{0} & 2 & \sqrt{3}-1 & -\sqrt{3}-1 \\
\mathbf{0} & 2 & 2 & -\sqrt{3}-1 & \sqrt{3}-1 \\
2 & 2 & \mathbf{0} & 2 & 2 \\
\sqrt{3}-1 & -\sqrt{3}-1 & 2 & \mathbf{0} & 2 \\
-\sqrt{3}-1 & \sqrt{3}-1 & 2 & 2 & \mathbf{0}
\end{array}\right]_{5,2}^{1}, \\
\frac{1}{21}\left[\begin{array}{rrrrr}
16 & \mathbf{0} & 10 & 4 \sqrt{5} & \sqrt{5} \\
\mathbf{0} & 5 & 4 & -4 \sqrt{5} & 8 \sqrt{5} \\
10 & 4 & \mathbf{0} & -7 \sqrt{5} & -4 \sqrt{5} \\
4 \sqrt{5} & -4 \sqrt{5} & -7 \sqrt{5} & \mathbf{0} & 6 \\
\sqrt{5} & 8 \sqrt{5} & -4 \sqrt{5} & 6 & \mathbf{0}
\end{array}\right]_{5,2}^{1}
\end{gathered}
$$

We give four examples for the next zero-pattern. The first example minimizes the square root, the second has the smallest denominator, the third minimizes the number of square roots, and the last one contains the smallest prime number under square root:

$$
\begin{gathered}
\frac{1}{245}\left[\begin{array}{rrrrr}
225 & 40 & 18 \sqrt{15} & -14 \sqrt{15} & \mathbf{0} \\
40 & 45 & -40 \sqrt{15} & \mathbf{0} & 180 \\
18 \sqrt{15} & -40 \sqrt{15} & \mathbf{0} & 175 & 6 \sqrt{15} \\
-14 \sqrt{15} & \mathbf{0} & 175 & \mathbf{0} & 42 \sqrt{15} \\
\mathbf{0} & 180 & 6 \sqrt{15} & 42 \sqrt{15} & -25
\end{array}\right]_{12}^{1}, \\
\frac{1}{108}\left[\begin{array}{rrrrr}
98 & 28 & 7 \sqrt{22} & -3 \sqrt{22} & \mathbf{0} \\
28 & 12 & -20 \sqrt{22} & \mathbf{0} & 44 \\
7 \sqrt{22} & -20 \sqrt{22} & \mathbf{0} & 42 & \sqrt{22} \\
-3 \sqrt{22} & \mathbf{0} & 42 & \mathbf{0} & 21 \sqrt{22} \\
\mathbf{0} & 44 & \sqrt{22} & 21 \sqrt{22} & -2
\end{array}\right]_{12}^{1},
\end{gathered}
$$




$$
\frac{1}{2527}\left[\begin{array}{rrrrr}
2457 & 12 \sqrt{195} & -420 & 380 & \mathbf{0} \\
12 \sqrt{195} & 343 & 80 \sqrt{195} & \mathbf{0} & -160 \sqrt{195} \\
-420 & 80 \sqrt{195} & \mathbf{0} & 2223 & 140 \\
380 & \mathbf{0} & 2223 & \mathbf{0} & 1140 \\
\mathbf{0} & -160 \sqrt{195} & 140 & 1140 & -273
\end{array}\right]_{12}^{1},
$$

In connection with Proposition 12, we raise the following special case of Conjecture 6 as a separate interesting problem.

Problem 19 For even $n$, show that there exists $X=X^{T} \in O_{n}(\mathbb{Q})$ with $\underline{X}=J_{n}-I_{n}$. For odd $n \geq 3$, show that there exists $X=X^{T} \in O_{n}(\mathbb{Q})$ with $\underline{X}=\Delta_{n, n-2}$ and $\operatorname{Tr}(X)=1$.

Many of the matrices in Section 2 and Section 3 have been constructed with the help of a computer. It is natural to raise the following problem:

Problem 20 Determine the computational complexity of the following decision problem:

- Given: A square (0,1)-matrix $M$ of size $n$.

- Task: Determine if $M$ is the zero-pattern of a real orthogonal matrix.

The size of $M$ gives the length of the input.

\section{References}

[1] L. B. Beasely, R. A. Brualdi and B. L. Shader, Combinatorial orthogonality, in Combinatorial and Graph-Theoretical Problems in Linear Algebra, R. A. Brualdi, S. Friedland and V. Klee, eds., Springer-Verlag, New York, pp. 207-218, 1993.

[2] G.-S. Cheon, C. R. Johnson, S.-G. Lee and E. J. Pribble, The possible numbers of zeros in an orthogonal matrix, Electron. J. Linear Algebra 5 (1999), 19-23.

[3] D. Ž. Djoković, K. Zhao, Near-diagonal representatives of conjugacy classes of orthogonal matrices, J. Algebra Appl., 7:5 (2008), 671-683.

[4] M. Fiedler, Symposium on the Theory of Graphs and Its Applications, Smolenice, Czechoslovakia, 1963. 
[5] M. Fiedler, Doubly stochastic matrices and optimization, Advances in mathematical optimization, 44-51, Math. Res., 45, Akademie-Verlag, Berlin, 1988.

[6] A. V. Geramita and J. Seberry, Orthogonal Designs: Quadratic Forms and Hadamard matrices, Marcel Dekker, New York, 1979.

[7] J. M. Goethals and J. J. Seidel, Orthogonal matrices with zero diagonal, Canad. J. Math., vol. 19, pp. 1001-1010 (1967).

[8] R. A. Horn and C. R. Johnson, Matrix Analysis, Cambridge University Press, Cambridge, UK, 1999.

[9] Y. Jiang, L. H. Mitchell, and S. K. Narayan, Unitary matrix digraphs and minimum semidefinite rank, Linear Algebra Appl. 428:7 (2008), 1685-1695.

[10] A. Landé, New Foundations of Quantum Mechanics, American Journal of Physics, Volume 34, Issue 12, pp. 1203-1204 (1966).

[11] J. D. Louck, Doubly stochastic matrices in Quantum Mechanics, Found. Phys. 27 (1997), no. 8, 1085-1104.

[12] R. Lundgren, K. B. Reid, S. Severini, and D. Stewart, Quadrangularity and strong quadrangularity in tournaments, Australas. J. Combin. 34 (2006), 247-260. arXiv:math/0409474v1 [math.CO]

[13] M. A. Nielsen and I. L. Chuang, Quantum computation and quantum information, Cambridge University Press, Cambridge, 2000.

[14] S. Severini, On the digraph of a unitary matrix, SIAM J. Matrix Anal. Appl., 25, 1 (2003), 295-300. arxiv:math.CO/0205187.

[15] S. Severini, F. Szöllősi, A further look into combinatorial orthogonality, Electron. J. Linear Algebra 17 (2008), 376-388. arXiv:0709.3651v1 [math.CO] 